\title{
Captura de mosquitos antropofílicos (Diptera, Culicidae) em uma área urbana da região oeste da Bahia, Brasil
}

\author{
Isana M. dos Santos \& Daniéla Calado
}

Universidade Federal do Oeste da Bahia, Centro das Ciências Biológicas e da Saúde, Programa de Pós-Graduação em Ciências Ambientais, Rua Professor José Seabra 316, Recanto dos Pássaros, 47808-021 Barreiras, Bahia, Brasil. (danielacalado@ufob.edu.br)

\begin{abstract}
Capture of anthropophilic mosquitoes (Diptera, Culicidae) in an urban area of the western Bahia, Brazil. The western region of Bahia undergoes accelerated agricultural development, which has generated infrastructure problems as the lack of sanitation and waste collection. Thus these problems play an important role in increasing the supply of breeding places of mosquitoes. Considering the possibilities of contacts between the vector and human population, the objective of this work was to study the adult Culicidae fauna in an urban area of western Bahia and to verify species of public health concern. The captures of adults were performed in Municipality of Barreiras during the period of February 2009 to January 2010, from 5:30 p.m. to 9 p.m. A total of 1,744 adults mosquitoes were capture and eight genera were identified: Aedes, Coquilletidia, Culex, Limatus, Mansonia, Ochlerotatus, Psorophora and Uranotaenia. The predominant species belong to Culex quinquefasciatus. Others species found with potential epidemiological importance were Aedes aegypti and Ochlerotatus scapularis. Whereas many species collected in Barreiras have epidemiological importance and occur even during the dry season due to lack of sanitation, it is important that municipalities of western Bahia be kept under constant epidemiological surveillance.
\end{abstract}

KEYWORDS. Mosquitoes, Culex, disease vectors.

RESUMO. A região oeste da Bahia passa por acelerado processo de desenvolvimento agrícola, o que tem gerado problemas de infraestrutura nos municípios. A falta de saneamento básico e de coleta dos resíduos são alguns desses problemas, os quais têm papel importante no aumento de criadouros disponíveis aos culicídeos. Assim, considerando as possibilidades de contato entre vetor e população humana, o objetivo deste trabalho foi estudar a fauna de culicíeos adultos em uma área urbana no oeste da Bahia e verificar a presença de espécies de interesse em saúde pública. As amostragens de mosquitos adultos foram realizadas no município de Barreiras, de fevereiro de 2009 a janeiro de 2010, das 17h30min às 21h00min. Ao total foram capturados 1.744 mosquitos e oito gêneros foram identificados: Aedes, Coquilletidia, Culex, Limatus, Mansonia, Ochlerotatus, Psorophora e Uranotaenia. Culex quinquefasciatus foi a espécie mais frequente e abundante. Outras espécies encontradas, e que apresentam importância epidemiológica, foram Aedes aegypti e Ochlerotatus scapularis. Considerando que muitas espécies coletadas em Barreiras possuem importância epidemiológica e ocorrem mesmo durante a estação seca devido à ausência de saneamento básico, é importante que os municípios da região oeste da Bahia sejam alvo constante das atividades de vigilância epidemiológica.

PALAVRAS-CHAVE. Mosquitos, Culex, insetos vetores.

As intensas modificações ocorridas em ambientes naturais têm favorecido o aparecimento de vários ecossistemas artificiais e o estabelecimento de condições favoráveis para o desenvolvimento e reprodução de culicídeos (Forattini, 1998). Essas modificações no ambiente podem alterar a dinâmica populacional de algumas espécies de mosquitos, colocando a população humana sob o risco de desenvolver doenças provocadas por agentes transmitidos por estes insetos.

$\mathrm{Na}$ região oeste do Estado da Bahia, a expansão da atividade agrícola tem provocado grandes alterações no Bioma Cerrado (Batistella \& Valladares, 2009), tais como: fragmentação de habitats, extinção da biodiversidade, invasão de espécies exóticas, poluição de aquíferos, degradação de ecossistemas e possivelmente modificações climáticas regionais. Somente no período de 1985 a 2005, houve acréscimo de 352\% na área plantada com culturas agrícolas e reduções substanciais de áreas naturais ocorreram nos municípios de Luís Eduardo Magalhães e Barreiras (SANo et al., 2011). Em decorrência desse desenvolvimento agrícola, muitos municípios da região oeste da Bahia passam por um acelerado processo de ocupação desordenado, o que tem acarretado problemas significativos relacionados à infraestrutura das áreas urbanas.

De acordo com LAPORTA et al. (2006), uma das causas do desenvolvimento de espécies de culicídeos epidemiologicamente importantes em áreas urbanas é o fato das cidades se desenvolverem carentes de saneamento, de educação e de saúde, contribuindo com o estabelecimento de condições favoráveis ao desenvolvimento de algumas espécies de mosquitos com importância em saúde pública. Além disso, Forattini (1998) também destacou que as mudanças ecológicas consequentes do desenvolvimento agrícola podem constituir importante fator no aparecimento de vetores emergentes, pois colocam o meio antrópico em contato mais estreito com espécies vetoras e os agentes infecciosos.

Apesar da realização de estudos sobre os culicídeos e sua ocorrência em áreas urbanas em território brasileiro, a diversidade desses insetos na região oeste do Estado da Bahia ainda é pouco conhecida. Assim, tendo em vista que o estudo das populações de culicídeos em centros urbanos fornece informações sobre a diversidade biológica e proporciona base para estudos epidemiológicos, objetivase identificar as espécies de mosquitos antropofílicos do 
município de Barreiras e contribuir com informações que possam ser utilizadas em programas/estratégias de controle de vetores na região oeste da Bahia.

\section{MATERIAL E MÉTODOS}

As coletas de culicídeos adultos foram realizadas no Campus Professor Edgard Santos da Universidade Federal do Oeste da Bahia (UFOB), localizado em área urbana do município de Barreiras. Este município está inserido no Bioma Cerrado no extremo Oeste da Bahia, entre $11^{\circ} 37^{\prime}-12^{\circ} 25^{\prime} \mathrm{S}$ e $44^{\circ} 34^{\prime}-46^{\circ} 23^{\prime} \mathrm{W}$. O clima da região é caracterizado por uma estação seca (maio a setembro) e outra chuvosa (outubro a abril), bem definidas. A precipitação pluviométrica média anual é de $1500 \pm 500$ $\mathrm{mm}$ e a temperatura média varia entre a mínima de $20^{\circ} \mathrm{C}$ e máxima de $26^{\circ} \mathrm{C}$ (Batistella \& Valladares, 2009).

$\mathrm{O}$ ponto das amostragens se localiza em área urbana

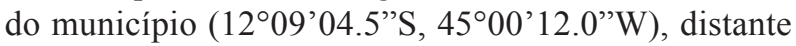
aproximadamente $200 \mathrm{~m}$ da margem direita do Rio Grande, afluente do Rio São Francisco. As áreas próximas a este ponto possuem vegetação de mata ciliar com diferentes fitofisionomias, variando de arbustiva à arbórea em diversos gradientes, e uma parcela significativa desse espaço já sofreu degradação pela ocupação humana.

Durante o período de fevereiro de 2009 a janeiro de 2010, amostras de culicídeos foram obtidas através da técnica pouso-homem segundo ForATtiNi et al. (2000), utilizando capturador de Castro. As amostragens ocorreram no horário das $17 \mathrm{~h} 30 \mathrm{~min}$ as $21 \mathrm{~h} 00 \mathrm{~min}$, sendo os culicídeos separados em intervalos de 30 minutos, compreendendo sete intervalos de coleta. Para evitar o "efeito intrusão", os coletores posicionaram-se no local de coleta 30 minutos antes do início do primeiro intervalo horário, ficando a uma distância de 1,5 m um do outro. O esforço amostral foi de 21 coletas, totalizando 77 horas.

A identificação dos espécimes foi realizada com auxílio de descrições e chaves dicotômicas (CONSOLI \& Lourenço-de-Oliveira, 1994; Forattini, 2002). Para identificação das espécies de Mansonia Blanchard 1901, lâminas da genitália feminina foram preparadas. Gêneros e subgêneros foram abreviados conforme REINERT (1975). Ochlerotatus foi tratado como gênero válido (REINERT, 2000) e abreviado segundo REINERT et al. (2004). As amostras estão depositadas na Coleção Zoológica da Universidade Federal do Oeste da Bahia.

Para as análises dos fatores abióticos, valores de temperaturas média, máxima e mínima $\left({ }^{\circ} \mathrm{C}\right)$, precipitação pluviométrica $(\mathrm{mm})$ e umidade relativa do ar (\%), foram obtidos do Instituto Nacional de Meteorologia (INMET) a partir da estação meteorológica automática de Barreiras $\left(12^{\circ} 09^{\prime} \mathrm{S}, 45^{\circ} 01^{\prime} \mathrm{W}\right)$, distante a menos de $3 \mathrm{~km}$ do local de captura. A partir desses dados foram calculadas médias retrospectivas para quinze e trinta dias anteriores à coleta, incluindo o dia da coleta. A relação entre os dados meteorológicos e o número de insetos capturados em cada coleta foi verificada através do Coeficiente de Correlação Linear de Pearson (alfa $=0,05$ ).

$\mathrm{Na}$ análise da atividade horária, foi considerado o número total de mosquitos por intervalo durante todo o período de coleta. Para a construção de gráficos e para as análises estatísticas empregou-se Microsoft Excel versão 2010 e BioEstat 5.0 (Ayres et al., 2007), respectivamente.

Tab. I. Espécies de Culicidae identificadas em área urbana do município de Barreiras, Bahia, Brasil no período de fevereiro de 2009 a janeiro de 2010.

\begin{tabular}{lc}
\hline Táxon & Total (\%) \\
\hline Tribo Aedini & $17(0,98)$ \\
Aedes (Stg) aegypti (Linnaeus, 1762) & $4(0,23)$ \\
Ochlerotatus serratus (Theobald, 1901) & $2(0,12)$ \\
Ochlerotatus stigmaticus (Edwards, 1922) & $184(10,63)$ \\
Ochlerotatus scapularis (Rondani, 1848) & $16(0,92)$ \\
Psorophora (Jan.) ferox (Humboldt, 1819) & $9(0,52)$ \\
Porophora (Jan.) varipes (Coquillett, 1904) & $1(0,06)$ \\
Psorophora (Jan.) sp. & $18(1,04)$ \\
Tribo Culicini & $1378(79,65)$ \\
Culex (Cux.) complexo coronator & $59(3,41)$ \\
Culex (Cux.) quinquefasciatus Say, 1823 & $14(0,81)$ \\
Culex (Cux.) spp. & $2(0,12)$ \\
Tribo Mansoniini & $7(0,40)$ \\
Mansonia (Man.) titillans (Walker, 1848) & $10,06)$ \\
Mansonia (Man.) indubitans Dyar \& Shannon, 1925 & $2(0,12)$ \\
Mansonia (Man.) sp. & $8(0,46)$ \\
Coquillettidia (Rhynchotaenia) venezuelensis (Theobald, 1912) & \\
Coquillettidia (Rhynchotaenia) lynch (Shannon, 1931) & $6(0,35)$ \\
Coquillettidia (Rhynchotaenia) sp. & $1(0,06)$ \\
Tribo Sabethini & \\
Limatus durhami Theobald, 1901 & $1(0,06)$ \\
Wyeomyia sp. & 1730 \\
Tribo Uranotaeniini & \\
Uranotaenia sp. & \\
\hline Total & \\
\hline
\end{tabular}




\section{RESULTADOS}

Durante o período de estudo, foram capturados 1.744 culicídeos. Deste total, 0,8\% foram representados por indivíduos machos, sendo excluídos das análises por não apresentam hábito hematófago. Na Tab. I são apresentados os táxons identificados, representados por oito gêneros pertencentes às tribos Culicini, Aedini, Sabethini, Mansoninii e Uranotaeniini. Culex quinquefasciatus Say, 1823 foi o táxon mais frequente e abundante, representou $79,6 \%$ do total das amostras coletadas e detectado em todas as coletas. Ochlerotatus scapularis (Rondani, 1848) $(10,6 \%)$ foi a segunda espécie mais numerosa, enquanto os demais táxons juntos representaram apenas $9,8 \%$ dos exemplares capturados.

Nas Figs 1-4 são apresentados o número total de culicídeos coletados e os dados referentes às variáveis meteorológicas do município de Barreiras. O período do inverno apresentou os mais baixos índices pluviométricos (junho 2,7 mm, julho $0 \mathrm{~mm}$ e agosto $0 \mathrm{~mm}$ ), compreendendo a estação seca no Cerrado. Durante os meses da primavera e do verão, se concentraram os mais elevados índices pluviométricos (estação chuvosa).

Os primeiros meses da estação chuvosa (novembro e dezembro) apresentaram maior abundância de culicídeos, entretanto, as análises de correlação entre o número culicídeos obtidos em cada coleta e os valores das variáveis meteorológicas, dos quinze e trinta dias que antecederam a coleta, não foram significativas. Os valores do coeficiente de correlação de Pearson obtidos com os dados referentes aos 15 dias anteriores à coleta foram: temperatura máxima $(r=-0,055)$, temperatura média $(r=$ $-0,019)$, temperatura mínima $(\mathrm{r}=0,247)$, umidade relativa do ar $(\mathrm{r}=-0,067)$ e precipitação pluviométrica acumulada $(\mathrm{r}=0,374)$. Para os 30 dias anteriores, foram obtidos os seguintes valores: temperatura máxima $(\mathrm{r}=0,135)$, temperatura média $(r=0,122)$, temperatura mínima $(r=$
0,017), umidade relativa do ar $(\mathrm{r}=-0,161)$ e precipitação pluviométrica acumulada $(r=0,078)$. Os meses onde não foi observada precipitação pluviométrica apresentaram menor riqueza, sendo observadas apenas $C x$. quinquefasciatus, Oc. scapularis (Figs 5, 6) e representantes de Mansonii, em número reduzido.

Quanto à atividade horária, o número de insetos capturados revelou tendência de acréscimo ao longo dos intervalos horários, com aumento evidente após o intervalo horário que compreendeu o crepúsculo vespertino (17h30min - 18h00min) (Fig. 7).

\section{DISCUSSÃO}

Apesar dos vários estudos realizados quanto à culicidofauna em território brasileiro (URBINATTI et al., 2001; Barbosa et al., 2003; Marcondes et al., 2003; Taipe-Lagos \& Natal, 2003; Hutchings et al., 2010; Morais et al., 2012), esta é a primeira lista de espécies para uma área urbana localizada no Cerrado baiano. Desta forma, os resultados aqui obtidos revestem-se de importância epidemiológica, já que alguns dos táxons identificados podem atuar na veiculação de patógenos e/ ou causar grande desconforto e reações alérgicas através da picada, promovendo a diminuição da qualidade de vida da população humana conforme já apontado por LOPES et al. (1995) e TEODORO et al. (1995) para áreas urbanas do sul do país.

Dentre os resultados obtidos destaca-se a elevada abundância de $C x$. quinquefasciatus ao longo de todo período estudado, mesmo em meses sem a ocorrência de chuvas. O valor de correlação obtido entre a precipitação pluviométrica acumulada e o número de mosquitos desta espécie (dados não mostrados) aponta que a presença de chuvas não é fator determinante para sua ocorrência e/ou para o aumento da densidade de indivíduos desta espécie. Isto sugere que as populações de culicídeos

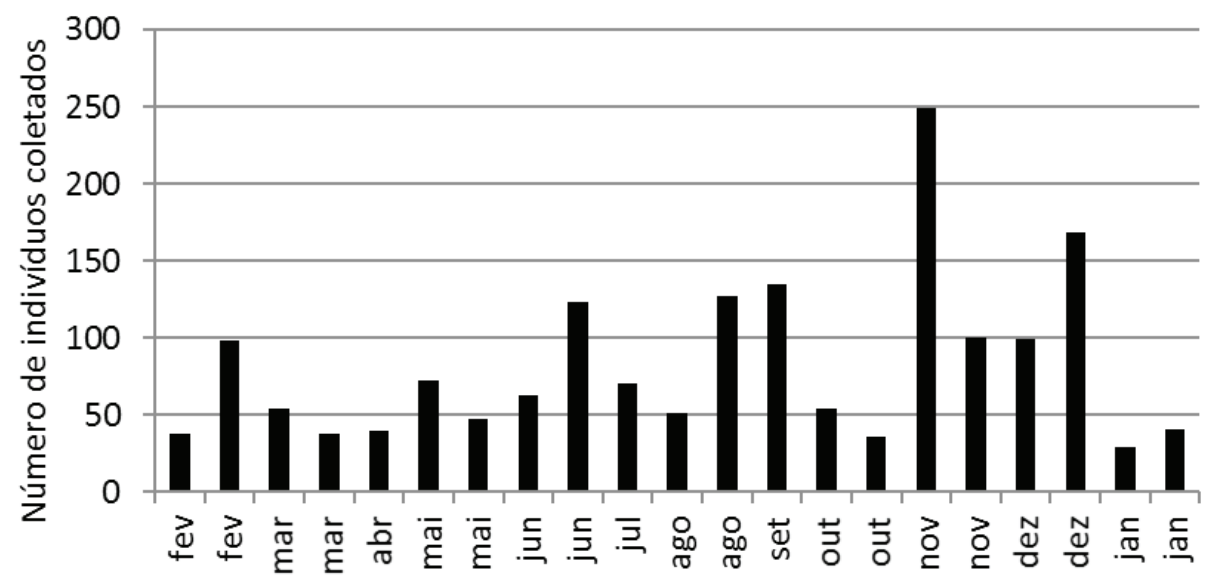

2009

2010

Fig. 1. Número total de culicídeos adultos coletados por coleta em área urbana do município de Barreiras, Bahia, Brasil no período de fevereiro de 2009 a janeiro de 2010. 

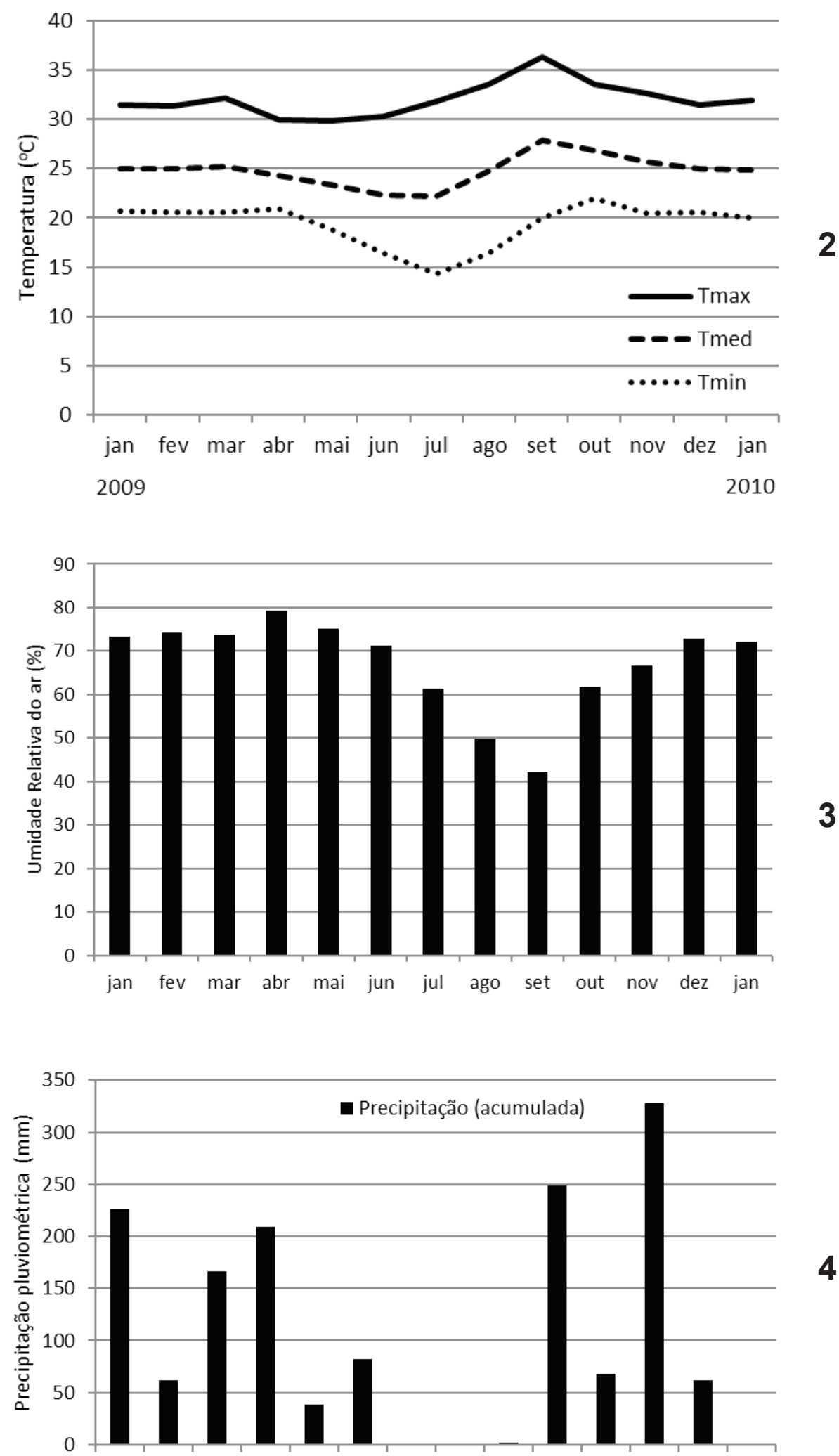

4

jan fev mar abr mai jun jul ago set out nov dez jan

Figs 2-4. Dados meteorológicos do município de Barreiras, Bahia, Brasil de janeiro de 2009 a janeiro de 2010: 2, umidade relativa do ar mensal (\%); 3, temperaturas máxima, média e mínima mensais (graus C); 4, precipitação pluviométrica acumulada (mm). 

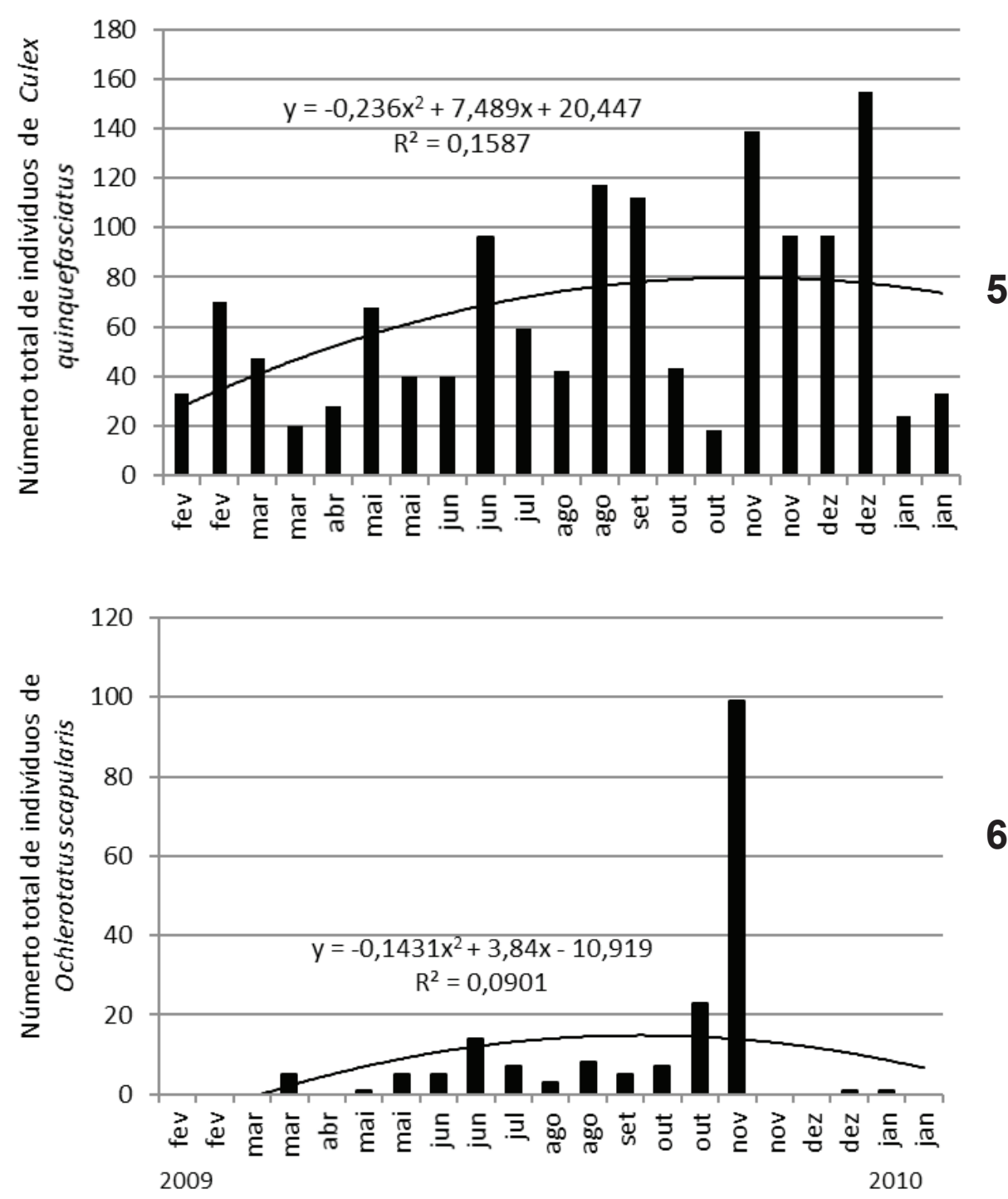

\section{6}

Figs 5,6. Número total de Culex quinquefasciatus (5) e Ochlerotatus scapularis (6) capturados em área urbana do município de Barreiras, Bahia, Brasil no período de fevereiro de 2009 a janeiro de 2010.

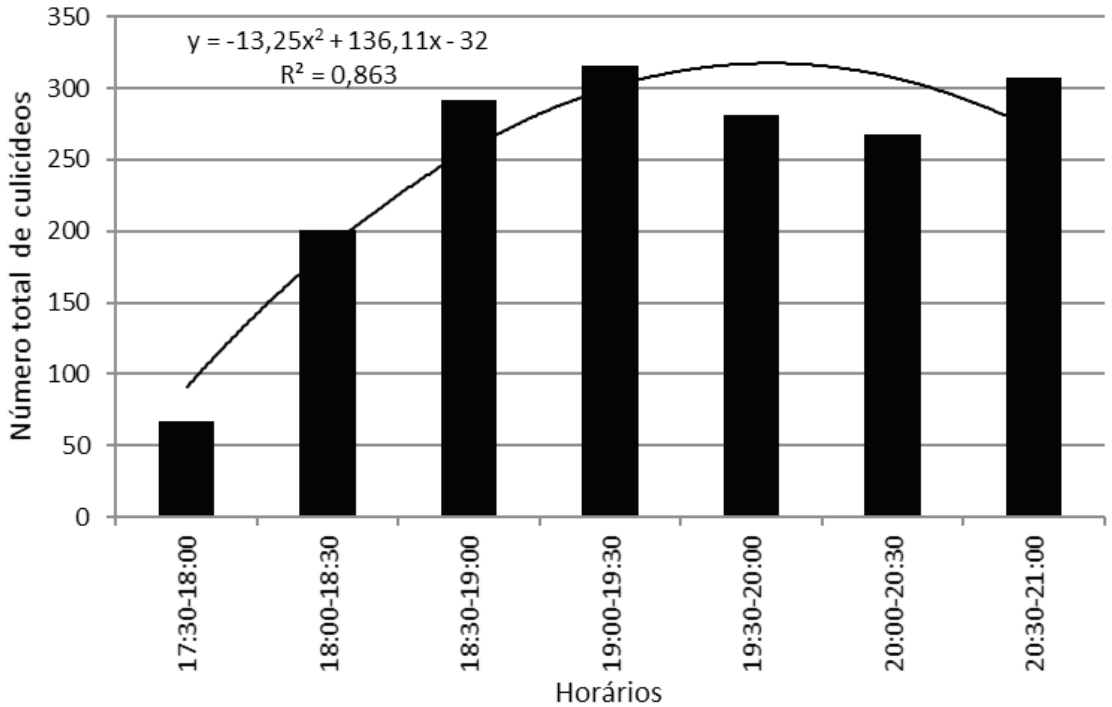

Fig. 7. Número total de culicídeos adultos capturados no intervalo das 17:30h as $21 \mathrm{~h}$ no município de Barreiras, Bahia, Brasil. 
na área de estudo também são influenciadas por outras condições ambientais, como por exemplo, a ausência de esgotamento sanitário e drenagem na maior parte do município. Estes fatores parecem promover a formação de múltiplos criadouros, os quais podem ser utilizados para o desenvolvimento das formas imaturas desta espécie mesmo durante a estação seca.

DAVID et al. (2012) também não verificaram correlação entre a densidade de indivíduos de $C x$. quinquesfaciatus e a variável precipitação pluviométrica. Estes autores apontam que, no município do Rio de Janeiro, a oferta de criadouros artificiais pode influenciar a ocorrência desta espécie, corroborando as informações obtidas no presente estudo para a região oeste da Bahia. Entretanto, comportamento diferenciado tem sido apontado para outras partes do mundo, como no estudo de UtтAн et al. (2013) realizado na Nigéria, onde a abundância da espécie segue o padrão das chuvas, tendo correlação positiva com a precipitação pluviométrica. Estas diferenças regionais demonstram que ainda é necessário o entendimento da biologia das populações de Cx. quinquefasciatus em diferentes cenários urbanos, e que as informações geradas podem auxiliar no controle dessa espécie conforme já destacado por DAVID et al. (2012).

Ainda quanto ao significado epidemiológico da elevada frequência e abundância de $C x$. quinquefasciatus, pode se destacar que esta espécie é o principal vetor da filariose brancoftiana no país. As regiões Norte e Nordeste do Brasil são consideradas endêmicas para filariose bancroftiana (MedeIRos et al., 2004), assim a ocorrência deste inseto em área urbana deve ser levada em consideração, especialmente porque a região oeste da Bahia apresenta intenso fluxo migratório, o que pode aumentar ainda mais o risco de ocorrência de doenças. Segundo o IBGE (2013), em 2010 a população do município era constituída por 137.427 habitantes dos quais aproximadamente $9 \%$ era representada por pessoas procedentes de várias regiões do país. Além do papel na transmissão de agentes de doença, TAipe-Lagos \& NATAL (2003) destacam ainda o incômodo que $C x$. quinquefasciatus pode causar para a população humana, em função da elevada antropofilia.

As demais espécies coletadas apresentam diferentes características quanto à ocupação de habitats e sua relação com a atividade humana. Segundo Dorvillé (1996), as espécies da Tribo Mansoniini, bem como Oc. scapularis, Aedes aegypti (Linnaeus 1762) e Cx. quinquefasciatus indicam ambientes com alto grau de antropização, enquanto algumas espécies de sabetíneos podem ser encontradas em ambientes silvestres (ForATtinI, 2002) e ambientes com alterações antrópicas não acentuadas (DoRvillé, 1996). A presença de espécies de Mansoniini na área urbana de Barreiras pode ser explicada pelo Rio Grande, que banha o município e que apresenta em alguns pontos quantidade considerável de plantas aquáticas (Eichornia crassipes (Mart.) Solms.), as quais podem ser utilizadas pelas formas imaturas - especialmente de Mansonia Blanchard, 1901.

Ochlerotatus scapularis foi registrado em maior número no mês de novembro, início da estação chuvosa. Por esta espécie ocupar criadouros no solo, a ocorrência de chuva no mês de outubro pode ter influenciado o aumento da população neste período através da maior oferta de criadouros. TAIPE-LAGos \& NATAL (2003) destacaram a adaptação desta espécie as áreas desmatadas e ForATtinI et al. (2000) revelaram ainda que a presença dessa espécie próximo ao espaço habitado e ao ambiente natural pode permitir a de transmissão de agentes infecciosos ao homem e animais domésticos, especialmente no caso das arboviroses.

O número reduzido de indivíduos de Ae. aegypti e Limatus durhami Theobald, 1901 pode estar relacionado ao hábito de vida das espécies e ao horário em que as coletas foram realizadas; Ae. aegypti é considerada espécie urbana e diurna, enquanto Li. durhami tem sido frequentemente coletada em áreas rurais e silvestres (LoPEs, 1999; Silva, 2002; Calderón-Arguedas et al., 2009) durante o dia. Outros táxons identificados neste estudo são característicos de ambientes florestais, tais como Wyeomyia Theobald, 1901 e Uranotaenia Belkin, 1953.

A coleta de espécies de hábito silvestre na área urbana de Barreiras pode ser justificada pela proximidade entre os ambientes urbano-rural-silvestre. No caso do município de Barreiras, as áreas próximas às margens do Rio Grande apresentam vegetação que pode contribuir para maior diversidade de espécies de culicídeos no centro urbano. BARBOSA et al. (2003) consideram que a preservação e a formação de ilhas de mata são estratégias importantes na estruturação do espaço urbano, porém o monitoramento destas áreas através de diferentes métodos de coleta de culicídeos deve fazer parte da rotina dos órgãos de vigilância epidemiológica.

A partir das informações obtidas neste estudo, sugere-se que nas áreas de Cerrado da região oeste da Bahia o controle de vetores deva levar em consideração a proximidade entre os ambientes silvestre-urbano-rural, carência dos serviços básicos de saneamento e drenagem urbana que geram potenciais criadouros, e a ocorrência de culicídeos mesmo na estação seca.

Agradecimentos. Ao Instituto Nacional de Meteorologia pelo fornecimento dos dados meteorológicos e à Fundação de Amparo à Pesquisa do Estado da Bahia (FAPESB) pelo auxílio financeiro e pela bolsa de Iniciação Científica a IMS.

\section{REFERÊNCIAS BIBLIOGRÁFICAS}

Ayres, M.; Ayres JR., M.; Ayres, D. L. \& Santos A. A. 2007. BioEstat: aplicações estatísticas nas áreas das ciências biológicas e médicas. Belém, Sociedade Civil Mamirauá, MCT-CNPq.

Barbosa, A. A.; Navarro-Silva, M. A. \& Calado, D. 2003. Atividade de Culicidae em remanescente florestal na região urbana de Curitiba (Paraná, Brasil). Revista Brasileira de Zoologia 20:59-63.

Batistella, M. \& Valladares, G. S. 2009. Farming expansion and land degradation in Western Bahia, Brazil. Biota Neotropica 9(3):61-76.

Calderón-Arguedas, O.; Troyo, A.; Solano, M. E.; Avendaño, A. \& BEIER, J. C. 2009. Urban mosquito species (Diptera: Culicidae) of dengue endemic communities in the Greater Puntarenas area, Costa Rica. Revista de Biologia Tropical 57:1223-1234.

Consoli, R. A. G. B. \& Lourenço-De-Oliveira, R. 1994. Principais 
mosquitos de importância sanitária do Brasil. Rio de Janeiro, Editora FIOCRUZ. 228p.

David, M. R.; Ribeiro, G. S. \& Freitas, R. M. 2012. Bionomics of Culex quinquefasciatus within urban areas of Rio de Janeiro, Southeastern Brazil. Revista de Saúde Pública 46(5):858-865.

DoRVILLÉ, L. F. M. 1996. Mosquitoes as bioindicators of forest degradation in southeastern Brazil, a statistical evaluation of published data in the literature. Studies on Neotropical Fauna and Environment 31:68-78.

Forattini, O. P. 1998. Culicidae mosquitoes as emerging vectors of diseases. Revista de Saúde Pública 32(6):497-502.

2002. Culicidologia Médica. São Paulo, Editora Edusp. v.2. 860p.

Forattini, O. P.; Kakitani, I.; Dos Santos, R. L. C.; Kobayashi, K.; Ueno, H. M. \& Fernandez, Z. 2000. Comportamento de Aedes albopictus e Ae. scapularis adultos (Diptera: Culicidae) no Sudeste do Brasil. Revista de Saúde Pública 34(5):461-467.

Hutchings, R. S. G.; Hutchings, R. W. \& SAllum, M. A. M. 2010. Culicidae (Diptera, Culicomorpha) from the western Brazilian Amazon: Juami-Japurá Ecological Station. Revista Brasileira de Entomologia 54(4):687-691.

IBGE - Instituto Brasileiro de Geografia e Estatística).2013.Cidades@. Disponível em: <http://www.ibge.gov.br/cidadesat/xtras/perfil. php? codmun $=290320 \&$ search $=$ Bahia $\mid$ Barreiras $>$. Acessado em: 27.06.2013.

Laporta, G. Z.; Urbinatti, P. R. \& Natal, D. 2006. Aspectos ecológicos da população de Culex quinquefasciatus Say (Diptera, Culicidae) em abrigos situados no Parque Ecológico do Tietê, São Paulo, SP. Revista Brasileira de Entomologia 50(1):125-127.

LOPES, J. 1999. Ecology of mosquitoes (Diptera, Culicidae) in natural and artificial rural breeding sites in northern Paraná State, Brazil. VIII. The influence of predator larvae (Toxorhynchites sp., Limatus durhamii and Culex bigoti) on the populations of Culex quinquefasciatus and Culex eduardoi. Revista Brasileira de Zoologia 16(3):821-826.

Lopes, J.; Oliveira, F. J. A. \& Oliveira, V. D. R. B. 1995. Predominância de Mansonia titillans (Walker, 1848) (Diptera: Culicidae) na área urbana do Município de Londrina - PR, sul do Brasil. Semina: Ciências Biológicas e da Saúde 16(2):254-259.

Marcondes, C. B.; Fernandes, A.; Paterno, U.; Müller, G. A.; Pinho, L. C. \& Struffaldi, D. 2003. New records of mosquitoes from the Southern states of Santa Catarina and Rio Grande do Sul, with 18 species new for the states (Diptera: Culicidae). Zootaxa 347:1-6.
Medeiros, Z.; Oliveira, C.; Quaresma, J.; Barbosa, E.; Aguiar-Santos, A. A.; Bonfim, C.; AlmeidA, J. \& Lessa, F. 2004. A filariose bancroftiana no município de Moreno - Pernambuco, Brasil. Revista Brasileira de Epidemiologia 7:73-79.

Morais, S. A.; Urbinatti, P. R.; Sallum, M. A. M.; Kuniy, A. A.; Moresco, G. G.; Fernandes, A.; Nagaki, S. S. \& Natal, D. 2012. Brazilian mosquito (Diptera: Culicidae) fauna. I. Anopheles species from Porto Velho, Rondônia state, western Amazon, Brazil. Revista do Instituto de Medicina Tropical de São Paulo 54(6):331-335.

REINERT, J. F. 1975. Mosquito generic and subgeneric abbreviations (Diptera: Culicidae). Mosquito Systematics 7:105-110.

2000. New classification for the composite genus Aedes (Diptera: Culicidae: Aedini), elevation of subgenus Ochlerotatus to generic rank, reclassification of the other subgenera, and notes on certain subgenera and species. Journal of American Mosquito Control Association 16:175-188.

Reinert, J. F; Harbach, R. \& Kitching, I. J. 2004. Phylogeny and classification of Aedini (Diptera: Culicidae), based on morphological characters of all life stages. Zoological Journal of the Linnean Society 142:289-368.

Sano, E.; Santos, C. C. M.; Silva, E. M. \& Chaves, J. M. 2011. Agricultural frontier of western Bahia: considerations about temporal and environmental aspect. Geociências 30:479-489.

SiLva, A. M. 2002. Imaturos de mosquito (Diptera, Culicidae) de áreas urbana e rural no norte do Estado do Paraná, Brasil. Iheringia, Série Zoologia 92:31-36.

TAipe-Lagos, C. B. \& NATAL, D. 2003. Culicidae mosquito abundance in a preserved metropolitan area and its epidemiological implications. Revista de Saúde Pública 37:275-279.

Teodoro, U.; Guilherme, A. L. F.; Lozovei, A. L.; La Salvia-Filho, V.; Funushigue, Y.; Sinosa, R. P.; Ferreira, M. E. M. C.; Barbosa, O. C. \& Lima, E. M. 1995. Culicidae of Itaipu lake, Paraná River, southern Brazil. Revista de Saúde Pública 29(1):6-14.

Urbinatti, P. R.; Sendaczb, S. \& Natal, D. 2001. Imaturos de mosquitos (Diptera: Culicidae) em parque de área metropolitana aberto à visitação pública. Revista de Saúde Pública 35(5):461-466.

Utтah, E. C.; Wokem, G. N. \& Okonofua, C. 2013. The Abundance and Biting Patterns of Culex quinquefasciatus Say (Culicidae) in the Coastal Region of Nigeria. ISRN Zoology. Disponível em: $<\mathrm{http}: / /$ dx.doi.org/10.1155/2013/640691>. Acessado em: 08.07.2013.

Recebido em 9 de julho de 2013. Aceito em 19 de março de 2014. ISSN 0073-4721

Artigo disponível em: www.scielo.br/isz 